\title{
Transcription factor neuromancer/TBX20 is required for cardiac function in Drosophila with implications for human heart disease
}

\author{
Li Qian ${ }^{a, 1}$, Bhagyalaxmi Mohapatra ${ }^{b}$, Takeshi Akasakaa , Jiandong Liu, ${ }^{a, 2}$, Karen Ocorra, Jeffrey A. Towbin ${ }^{b}$, \\ and Rolf Bodmer ${ }^{\mathrm{a}, 3}$ \\ aDevelopment and Aging Program, Del E. Webb Neuroscience, Aging and Stem Cell Research Center (NASCR), Burnham Institute for Medical Research, \\ 10901 North Torrey Pines Road, La Jolla, CA 92037; and 'Pediatric Cardiology, Baylor College of Medicine, Texas Children's Hospital, Houston, TX 77030
}

Edited by Eric N. Olson, University of Texas Southwestern Medical Center, Dallas, TX, and approved October 16, 2008 (received for review September 3, 2008)

\begin{abstract}
neuromancer/Tbx20 ( $\mathrm{nmr}$ ) genes are cardiac T-box transcription factors that are evolutionarily conserved from flies to humans. Along with other known congenital heart disease genes, including tinman/ $N k x 2-5$, dorsocross/Tbx5/6, and pannier/Gata4/6, they are important for specification and morphogenesis of the embryonic heart. The Drosophila heart has proven to be an excellent model to study genes involved in establishing and maintaining the structural integrity of the adult heart, as well as genes involved in maintaining physiological function. Using this model, we have identified $n m r$ as a gene required in adult fly hearts for the maintenance of both normal myofibrillar architecture and cardiac physiology. Moreover, we have discovered synergistic interactions between $\mathbf{n m r}$ and other cardiac transcription factors, including tinman/Nkx2-5, in regulating cardiac performance, rhythmicity, and cardiomyocyte structure, reminiscent of similar interactions in mice. This suggests a remarkably conserved role for this network of cardiac transcription factors in the genetic control of the adult heart. In addition, nmr-tinman interactions also influence the expression of potential downstream effectors, such as ion channels. Interestingly, genetic screening of patients with dilated cardiomyopathy and congenital heart disease has revealed TBX20 variants in three sporadic and two familial cases that were not found in controls. These findings suggest that the fly heart might serve as an identifier of candidate genes involved in human heart disease.
\end{abstract}

arrhythmia | cardiac disease | T-box genes | tinman

T he Drosophila homeobox transcription factor (Tinman) was the first key determinant of heart development identified in the animal kingdom (1-3). Its vertebrate homolog, Nkx2-5, was subsequently identified in vertebrates. Since then, the identified components that make up the transcriptional network controlling heart formation have become increasingly complex. The factors that guide cardiac specification and differentiation in the fly appear to be highly conserved across species and now include a number of homeodomain, GATA, MADS-box, and T-box factors (3-6). Although much progress has been made in determining the roles of these factors during cardiac development, their roles in adult heart function remain to be elucidated.

The T-box transcription factor Tbx20 is a member of a family of ancient T-box genes related to the Tbx1 subfamily (7). Studies of Tbx20 in vertebrate models indicate a critical role in the induction and support of a core cardiac transcription factor network (8-11). For example, in Tbx20 knock-out mice, the expression of other cardiac transcription factors is downregulated or delayed, similar to observations in flies with mutations in the Tbx20-related neuromancer genes (nmrl\&2; also known as H15 and midline, respectively) (12-14). Mutations in Tbx5, Nkx2-5, and Gata4 cause a range of congenital heart diseases (CHDs) including atrial-septal and conduction defects (15-19). Several findings suggest that Tbx20 is also likely to be involved in CHD. Tbx20 directly interacts with these cardiac transcription factors $(10,20,21)$, but its role in adult heart function is not known because $T b \times 20$ knock out mice are embryonic lethal and conditional heart-specific manipulations have not been done as of yet. Thus, the importance of $T b \times 20$ interactions for adult heart structure and function in vivo needs to be further clarified.

Drosophila models are increasingly popular for studying a number of postnatal human diseases including cancer, diabetes, or neurodegenerative diseases $(22,23)$. Recently, Drosophila has also become a unique and well-suited genetic model for studying potential heart disease genes $(6,22,24-26)$, including the cardiogenic NKX, GATA and TBX transcription factors. For example, tinman/Nkx2-5 function in both flies and mice has been shown to be required not only for embryonic cardiogenesis $(1,2,27,28)$ but also for adult heart morphology and function $(29,30)$.

In this study, we use image-based cardiac contraction analysis and electrical pacing-induced cardiac stress tests $(26,31)$ to examine the role of $n m r / T b \times 20$ in the adult Drosophila hearts. Using the TARGET system (32) to spatially and temporally manipulate gene expression in the adult fly heart, we find that this cardiac transcription factor is also required in the adult for maintaining heart function. In addition, we identify a genetic interaction between $\mathrm{nmr}$ and tinman and show that this interaction is required for cardiac morphology, performance, and heartbeat rhythmicity. These studies demonstrate that Drosophila has potential as a postnatal model system for exploring the genetics underlying heart disease. As a first step in evaluating this potential, human subjects with structural congenital heart disease, as well as heart muscle dysfunction were examined. In 96 human subjects with clinical evidence of dilated cardiomyopathy (DCM), as characterized by left ventricular dilation and systolic dysfunction, DNA analysis identified three different variants of TBX20 in individuals with sporadic and familial DCM cases, suggesting TBX20 gene may be involved in the development of cardiomyopathy. In addition, TBX20 variants were identified in four children with atrial septal defect (ASD) with or without additional congenital heart defects. This underlines the potential utility of genetic screening in Drosophila as a discovery tool for candidates in human cardiovascular disease.

\section{Results}

Expression of $\mathbf{n m r}$ in Adult Cardiomyocytes. $\mathrm{nmr}$ genes are prominently expressed in the embryonic myocardium (12-14). To 

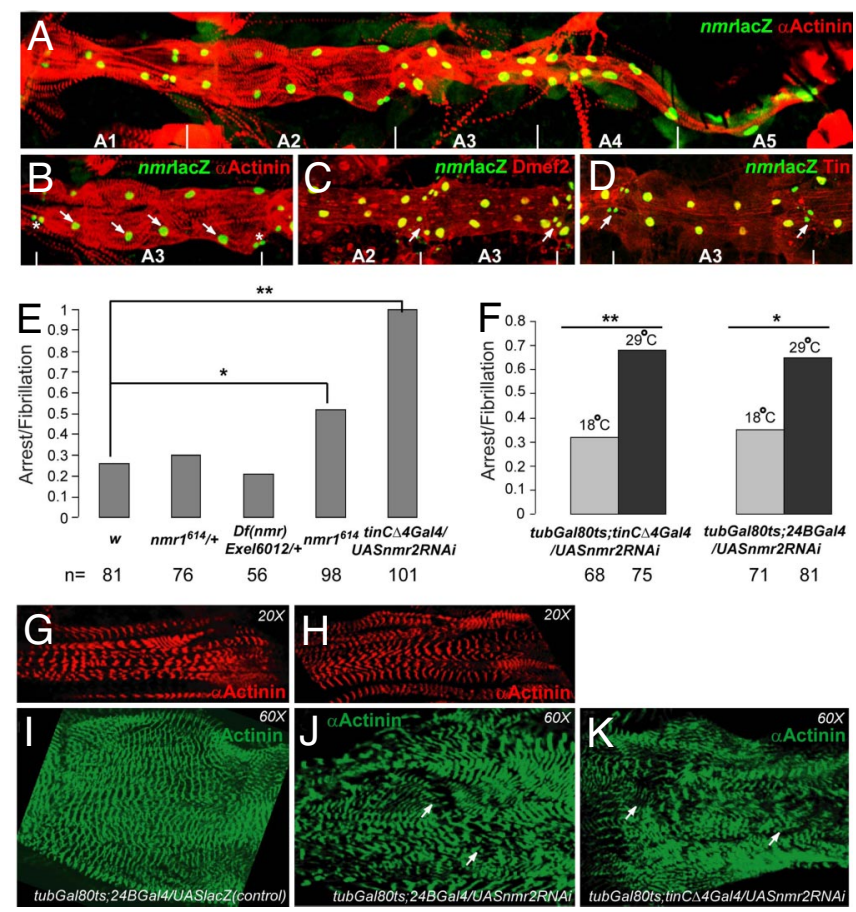

7181

Fig. 1. Requirement for $n m r / T b x 20$ in adult heart function. ( $A$, and $B$ ) Double labeling of $n m r$-lacZ ( $n m r 1, H 15)$ with myofiber marker $\alpha$-Actinin shows nuclear $\mathrm{nmr}$ expression in four Tinman-positive cardiomyocytes (arrows in $B$ ) and two Seven-up-positive ostia cells (asterisks in $B$ ) in each hemisegment. (C) Double labeling for $n m r$-lacZ and muscle-specific transcription factor Dmef2 indicates co-localization of $\mathrm{nmr}$ and Dmef2 in all myocardial cells including ostiae cells (arrows). (D) Double labeling of $n m r$-lacZ with Tinman cardiomyocyte nuclei. Notice that Tinman is absent in the ostia (arrows). (E) Loss of $n m r 1$ or cardiac knock-down of $n m r 2$ results in a dramatic increase in heart arrest/fibrillation rate (percentage of flies whose hearts failed when paced, see Materials and Methods). $\chi^{2}$ analysis compared to wild type $(w)$ and controls ( $n m r 1614 /+$ and $\operatorname{Df}(2 \mathrm{~L})$ Exel612/+): *, $P<0.01, * *, P<0.001, n=50-100$. ( $F$ ) TARGET-mediated knock-down of $n m r 2$ in the adult myocardium after eclosion results in increased arrest/fibrillation rate upon pacing stress $\left(29^{\circ} \mathrm{C}\right)$ compared to uninduced controls $\left(18^{\circ} \mathrm{C}\right)$. See Table S1 for details of statistical analysis. $(G-K)$ $\alpha$-Actinin labeling of the adult heart (one segment). ( $G$ and $H$ ) Confocal sections through the outer longitudinal heart-associated muscle layer indicate no difference between $n m r 2-k o c k d o w n$ hearts and controls. (I-K) Sections through the inner cardiomyocytes show abnormal arrangement of the spiral myofibers (arrows) in $n m r 2$ adult specific mutant hearts using either the $24 B$-Gal4 $(J)$ or $\operatorname{tin} C \Delta 4-G a l 4$ driver $(K)$.

determine whether cardiac expression persists, we examined $\mathrm{nmr}$ expression in the adult heart. Double labeling with $n m r^{\mathrm{H} 15}$-lacZ (13), and $\alpha$-Actinin (33), a Z-line marker of myofibers, shows that $n m r$ is expressed in the adult heart and co-labels with Dmef2 and Tinman-positive nuclei of the contractile myocardium (Fig. $1 A-D)(34)$. The two smaller Dmef2-positive nuclei correspond to the ostiae, which form openings in the heart tube controlling movement of the hemolymph into and out of the heart (asterisk in Fig. $1 B$ and arrows in Fig. $1 C$ and $D$ ). This observation is consistent with our real time quantitative RT-PCR results in which $n m r 1 \& 2$ mRNA were both detected in the adult fly heart (see Fig. 4), indicating $n m r$ genes are indeed expressed in adult heart.

Pacing-Induced Heart Arrest/Fibrillation in nmr/Tbx20 Mutants. Since tinman is expressed and required in the adult heart, we wondered whether $n m r$ also contributes to adult cardiac structure and function. $n m r 1$ null mutants $\left(n m r 1^{614}\right)$ are viable and show a mild embryonic heart phenotype, whereas mesodermal $n m r 2$ knockdown is almost as severe a (cardiac) phenotype as $n m r 1 \& 2$ double mutants (12-14). To test the roles of these genes in adult flies, we measured cardiac performance in $\mathrm{nmr}$ mutants using an electrical pacing protocol, which stresses the heart and induces fibrillation or arrest, especially in aged or compromised hearts (31). Although we have previously used the term "heart failure" to describe this cardiac dysfunction $(24,31)$, this term does not imply the "pump failure" used to describe mammalian heart function but rather an alteration or cessation of normal cardiac pumping as a result of the pacing induced stress. First, we examined flies heterozygous for $n m r 1^{614}$ or a larger deficiency covering both $n m r 1 \& 2$, $\operatorname{Df}(2 \mathrm{~L})$ Exel6012. No significant increase in heart arrest/fibrillation following pacing was observed in these flies compared to wild type (Fig. $1 E$ and supporting information (SI) Table S1). However, when both copies of $n m r 1$ are removed $\left(n m r 1^{614}\right)$ or when $n m r 2$ function is reduced with heart-specific knockdown (tinC $\Delta 4$-Gal4 driving UAS-nmr2-RNAi) (13), there is a dramatic increase in the rate of pacing-induced heart arrest/fibrillation (Fig. $1 E$ and Table S1), which further increases with age (Fig. S1 $A$ and $D$ ). The downregulation of $n m r 2$ in the heart also results in a severely truncated lifespan (Fig. S1B), similar to what is observed for flies with a cardiac-specific loss of tinman expression (30). To distinguish between an embryonic versus an adult requirement for $n m r$ we knocked down $n m r 2$ in the heart only during the adult stage (using the TARGET system (32): $t u b$-Gal80-ts;tinC $\Delta 4$ Gal4 or $t u b$-Gal80ts;24BGal4 crossed to UAS-nmr2-RNAi, see Materials and Methods). In these flies, we also observed an increased rate in pacinginduced heart arrest/fibrillation compared to control flies (Fig. $1 F$, Fig. S1D, and Table S1). Although we cannot categorically rule out that some RNAi off-target effects may contribute to observed phenotype, these data strongly suggest that $n m r$ function is indeed required for normal cardiac performance in adult flies. This conclusion is further substantiated by the effects on cardiac function and morphology as a result of genetic interactions between $\mathrm{nmr}$ deficiencies and tinman (see Fig. 3).

Arrhythmias and Abnormal Myofibrillar Structure in nmr/Tbx20 Mutants. We conducted image-based contraction analysis in $\mathrm{nmr}$ mutant flies. High-speed movies were taken from dissected and exposed hearts and contractions were displayed as M-modes (see Materials and Methods) showing the dynamics of heart wall movements over time (26). Heart specific knock-down of $n m r 2$ causes a considerably irregular beating pattern and an overall increase in the heartbeat length compared to the controls (Fig. $2 A-C^{\prime}$ ). These arrhythmias are due to the increased variability of diastolic (but not the systolic) interval lengths of the heart period (Fig. $2 A-F$ ). The severity of arrhythmia is quantified by the standard deviation of the mean heart period for each fly ("arrhythmia index," Fig. $2 F$; see ref. 26). Thus, $n m r$ mutant hearts not only beat more slowly but are also arrhythmic. Depleting $n m r$ levels in the adult heart possibly accelerates the cardiac aging process, since both the incidence of arrhythmias and length of the heart period normally increase with age (26).

Given the heart function defects observed for $n m r$ mutants, we were also interested in examining these hearts for cellular defects. During metamorphosis, the fly heart undergoes a remodeling process producing a regular spiral or transverse muscle fiber pattern within the tinman-expressing myocardial cells (see Fig. $1 A-D$ ). In addition, a new set of longitudinal fibers that express Dmef 2 but not tinman forms ventral to the heart (Fig. $1 C$ and $G)(34,35)$. We examined $n m r 2$ knockdown hearts using $\alpha$-Actinin antibodies, which localize to Z-lines of Drosophila muscle fibers (33). Using this marker we find that the regularity of the myofibrillar alignment and the spacing between Z-lines is disrupted within the Tinman-positive myocardium, where $n m r 2$ expression is diminished (Fig. $1 I-K$ and Fig. $\mathrm{S} 1 E$ ), but not in the ventral longitudinal muscle fibers that do not express tinman (Fig. $1 G$ and $H$ and Fig. S1E). No noticeable change in cell death is observed; however, death in 1-2 cells per heart could possibly contribute to the observed myofibrillar disarray. Changes in cardiomyocyte size can also be estimated from measurements of diastolic diameter (DD) and systolic diameter 
A

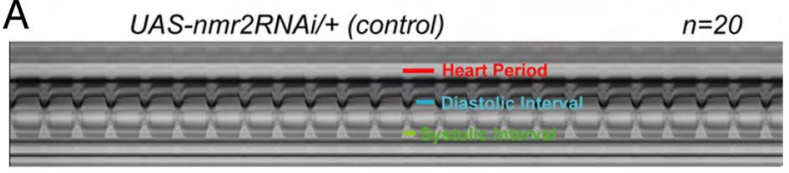

$\mathrm{B}$

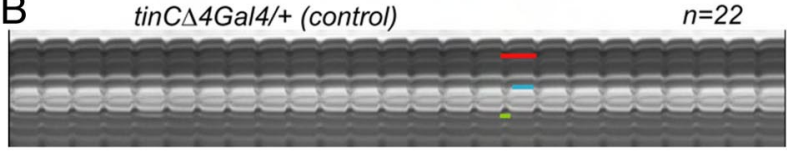

C
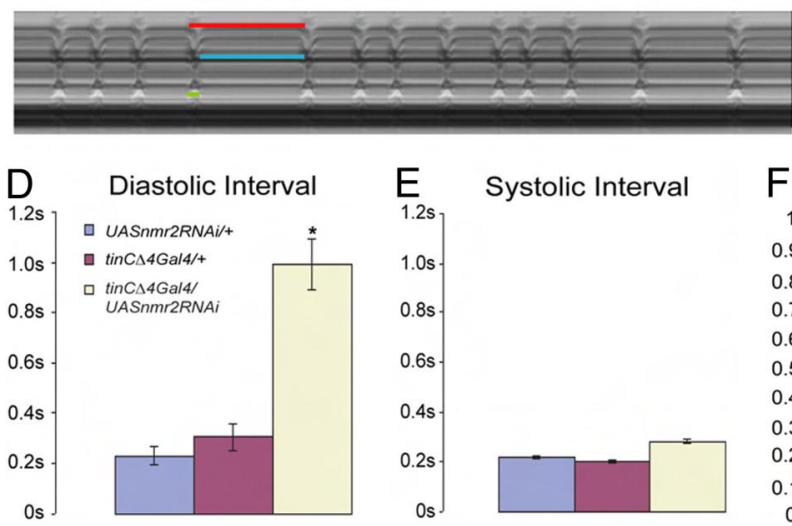
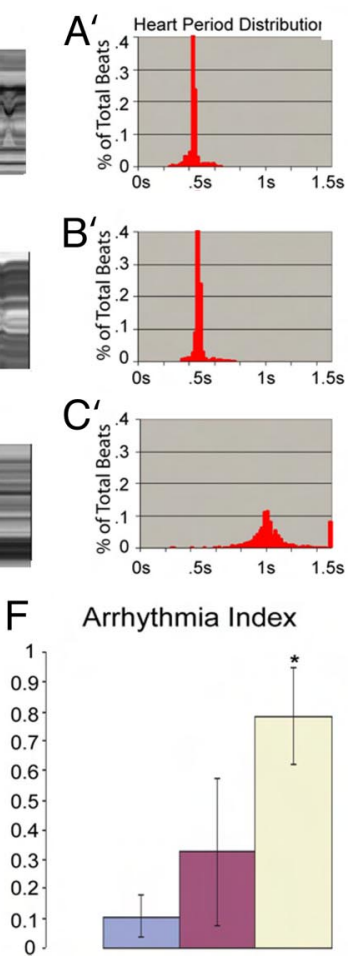

Fig. 2. Arrhythmias in $n m r$ mutant adult hearts. (A-C) M-mode traces prepared from high-speed movies of semiintact flies (for methods see Ocorr, et al. in ref. 26). ( $A$ and $B)$ M-modes from control flies show regular beating pattern. (C) Arrhythmic heart beats are evident in cardiac $n m r 2$ knockdown flies. Representative traces are shown. $\left(A^{\prime}-C^{\prime}\right)$ Histograms of heart periods (method as described in ref. 26). No differences were observed between males and females of each genotype that were pooled ( $n$ as indicated above each M-mode). ( $D-F$ ) Statistical analysis of heart contraction in $n m r 2$ adult specific mutant hearts. $n m r 2-R N A i$ expression in the heart results in slower heart beat due to longer Diastolic Intervals $(D)$ and in a dramatic increase in the incidence of arrhythmias $(F)$, without affecting the Systolic Interval (E).
(SD) of the heart tube (Fig. S1 $F$ and $G$ ). We found a moderate decrease in DD and a mild increase in SD for $n m r 2$ adult-specific knockdown mutants. These two changes result in a significant decrease in fractional shortening compared to controls, indicating a change in contractility in $n m r$ knockdown hearts (Fig. S1H). The observed abnormalities in myofibrillar structure in response to decreased $n m r$ expression likely contribute to these functional defects.

Genetic Interaction Between nmr/Tbx20 with tinman/Nkx2-5 in Regulating Adult Heart Function. One of the enduring problems in the study of cardiac disease loci is the relative contribution of the genetic background or polygenic interactions that may ameliorate or aggravate the phenotypic expression of genetic abnormalities. This is the case for known cardiac disease loci, such as Nkx2-5, Tbx5, and Gata4, which cause a phenotype as heterozygotes $(15-18,36)$. Therefore, we examined whether the fly's T-box and Nkx homologs interact genetically to control adult heart physiology. We combined the heterozygous nmr1\&2 deficiency $\operatorname{Df}(2 \mathrm{~L})$ Exel6012 with heterozygotes for either tinman or $\operatorname{Df}(3 \mathrm{~L}) D o c A$ (which span all three Drosophila Tbx5/6 related genes) $(14,37)$. The pacing-induced heart arrest/fibrillation rate for $\operatorname{Df}(2 \mathrm{~L})$ Exel6012/+;tinman/+ or Df(2L)Exel6012/ $+; \operatorname{Df}(3 \mathrm{~L}) D o c A /+$ is dramatically elevated compared to the single heterozygous controls (Fig. $3 A$ and Table S1). This phenotype could be rescued by expressing wild-type $n m r 2$ cDNA specifically in the heart (Fig. $3 B$ and Table S1), indicating that the observed phenotype is indeed due to the genetic interaction of tinman or the $d o c$ genes with the $n m r$ locus. An interaction of $\operatorname{Df}(2 \mathrm{~L})$ Exel6012 $2^{+/-}$with tinman $^{+/-}$was also observed with respect to the development of arrhythmias (Fig. $3 C-E$, Fig. S2 $A-E)$, the level of cardiac expression of potential downstream effector genes (Fig. 4), and the alignment of myofibers within the cardiac myocytes (Fig. $3 F-H$ ). The genes whose expression is most affected as a result of tinman-nmr interactions are the $n m r$ genes themselves, a potassium channel, an ATPase (SERCA), and dystrophin, all of which are likely to be involved in heart morphogenesis or function (Fig. 4). Indeed a dystrophin deficiency has previously been shown to cause myofibriller misalignment in the fly heart
(38). Whether the observed changes in gene regulation are direct, indirect, or the consequence of a compensatory mechanism remains to be examined. Nevertheless, our data suggest that cardiogenic transcription factors interact strongly in establishing and maintaining adult heart structure and function.

Cardiac performance exhibits a progressive deterioration with age, which is manifest by an increase in pacing-induced heart arrest/fibrillation rate and in arrhythmias $(24,26)$. Since tinman and $n m r$ significantly influence adult heart function, we wondered whether reduced cardiac performance in old flies could be rescued by overexpression of these factors. When $n m r 2$ is overexpressed beginning at the 5 th week of adult life (Fig. S2F; see Materials and Methods), pacing-induced arrest/fibrillation rate is significantly reduced compared to non-overexpressors (Fig. S2 $G$ ). Similar effects were observed with tinman cDNA overexpression in aging fly hearts (Fig. S2H). This suggests that maintaining high levels of tinman or $n m r$ expression in older hearts halts or slows the age-dependent cardiac performance decline.

TBX20 Mutations in Humans with Dilated Cardiomyopathy. Since most, if not all, transcription factors with a demonstrated role in the fly heart also function in vertebrates, we wondered whether $T B X 20$ sequence variants could be associated with cardiomyopathies in human patients. For this purpose, unrelated probands with clinically apparent dilated cardiomyopathy (DCM), who presented with echocardiographic evidence of left ventricular (LV) dilation, systolic dysfunction, and clinical evidence of heart failure and cardiac rhythm disturbance were enrolled for this study. All subjects and controls had blood drawn, DNA was isolated, and lymphoblastoid cell lines were developed. Patient $(n=96)$ and control $(n=392)$ DNA samples were screened for non-synonymous variants (mutations) in TBX20 and NKX2-5 genes by direct DNA sequencing (for primers see Table $\mathrm{S} 2$ ). This revealed three missense mutations (L196V, R334Q and W349R) in the TBX20 gene in Caucasians, two of which occurred with incomplete penetrance in familial cases (see Fig. 5 and Fig. S3). None of these mutations were identified in 392 ethnic-matched controls (nearly 800 chromosomes; see Table S1). Two of the variants had alterations in conserved residues in the 

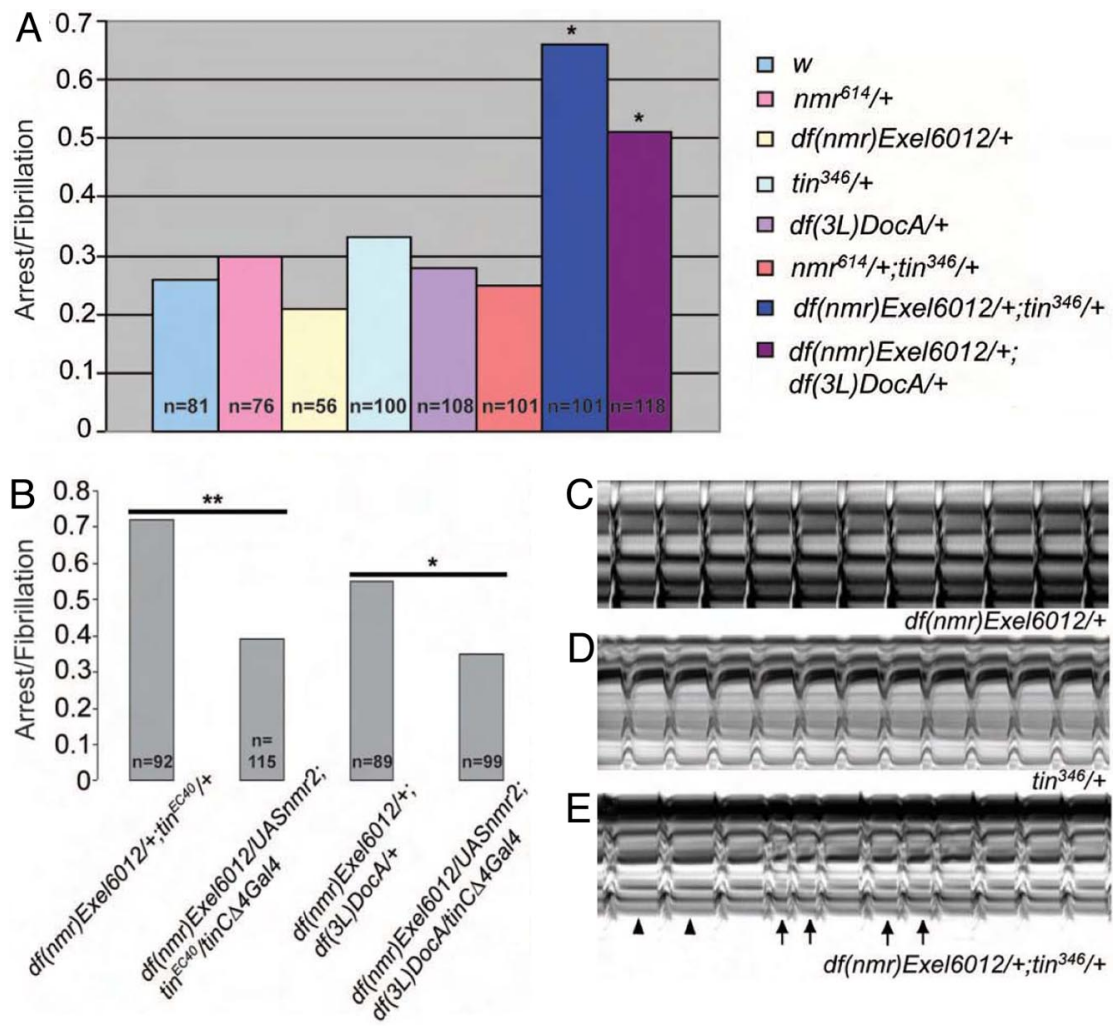

Fig. 3. Abnormal cardiac function and morphology in $n m r$, tin transheterozygotes. (A) Heart arrest/fibrillation rates of transheterozygous combinations of neuromancer/Tbx20 (Df(2L)Exel6012) with tinman ( $\operatorname{tin}^{340}$ ) or dorsocross/Tbx5/6 ( $\mathrm{Df}(3 \mathrm{~L}) D o c A)$, and corresponding controls. Sample sizes are 50-100 flies/data point. $\chi^{2}$ analysis [with respect to all controls; see Wessells, et al. (2004) in ref. 24 for details]: *, $P<0.01$ (see Table $S 1$ for individual values). (B) Increased heart arrest/fibrillation rate in transheterozygotes of $n m r$ and tinman or doc is rescued by expressing $n m r 2$ CDNA in the heart. ( $\chi^{2}$ analysis: $*, P<$ $0.01 ; * *, P<0.001$, see Table $S 1$ for individual values). $(C-E)$ Representative M-mode traces from each genotypes. Note the increased incidence of arrhythmia in $n m r+l-; t i n+l-$ flies (arrows in $H$ indicate shortened heart beats, arrowheads in $H$ show heart beats with longer diastolic interval). ( $F$ and $G$ ) $\alpha$-Actinin labeling of adult heart. As compared to control $(n m r+l-, F)$, $n m r+l-;$ tin $+l-(G)$ transheterozygous mutants show disruption in the regular myofibril alignment and Z-lines spacings. $(H)$ Quantification of myofiber irregularities. Severity is determined by the average number of segments ( 5 in total as shown in Fig. $1 A$ ) with significant abnormal myofiber structure (as in $G$ ). Student $t$ test: *, $P<0.05$.

DNA-binding T-box of TBX20 (Fig. S3). Notably, variants in the familial cases of DCM demonstrated reduced penetrance, which is a common finding in inherited forms of cardiomyopathy. In Family 4 , for instance, only half of the individuals with the R334Q variant had clinically apparent DCM at the time of the evaluation. All non-synonymous variants found in patients and ethnic-matched controls are listed in Tables S3 and S4. No potentially diseasecausing mutations in NKX2-5 were identified in the examined patient cohort and no affected subject lacked the variant in family 4. These findings imply that candidate gene functions found in the Drosophila model are potentially relevant for human cardiac disease. However, definitive causality cannot be claimed at this point, in part because of the sporadic or incompletely penetrant nature of the cases identified. Although this is a common observation in human cardiovascular diseases such as cardiomyopathies and arrhythmia disorders $(40,41)$, it limits our ability to conclude a cause-and-effect relationship at this time. To demonstrate a causal relationship between gene mutation and disease, it will be important to conduct linkage analysis in large families with identified and functional studies of the identified variants. Nevertheless, our observations of a number of patients (but not controls) with non-synonymous variations in conserved regions of the coding region of $T B X 20$ make this gene a promising candidate to potentially be involved in the human disease.

Role of TBX20 in the Genetics of Congenital Heart Disease in Humans. Kirk, et al. previously reported that, in addition to DCM, mutations in TBX20 are also associated with ASD and mitral valve disease
(41). Consequently, we screened children with CHD including ASDs and ventricular septal defects (VSDS), as well as more complex CHD associated with these septal defects. In addition, mitral valve disease was evaluated. We screened 96 children with ASD and 96 with VSD associated disease. In four children, sporadic TBX20 variants were identified. A TBX20 variant in exon 4 resulting in a $597 \mathrm{C}>\mathrm{G}$ alteration, which changes a histidine to an aspartic acid (H186D), was found in two unrelated children. In one child, an African American female, an atrioventicular canal with secundum and primum ASDs, as well as a cleft mitral valve with moderate mitral regurgitation was noted. The second child, a Hispanic male, had pentalogy of Fallot (tetralogy of Fallot with ASD). The other exon 4 mutation $(601 T>C$; L197P) was identified in an African American female with pentalogy of Fallot and in a white female with isolated ASD. None of these variants were identified in 392 ethnic-matched controls (see Tables S3 and S4).

\section{Discussion}

Drosophila has been successfully used to study the regulatory genetic networks of inductive signals and transcription factors that determine cardiac specification and morphogenesis, but the genetic control of cardiac physiology is only beginning to be investigated. In this study, we used the Drosophila model to identify novel roles for the T-box transcription factor $n m r / T b \times 20$ and the homeobox gene tinman in establishing cardiac function and maintaining the structural integrity of the adult heart. 

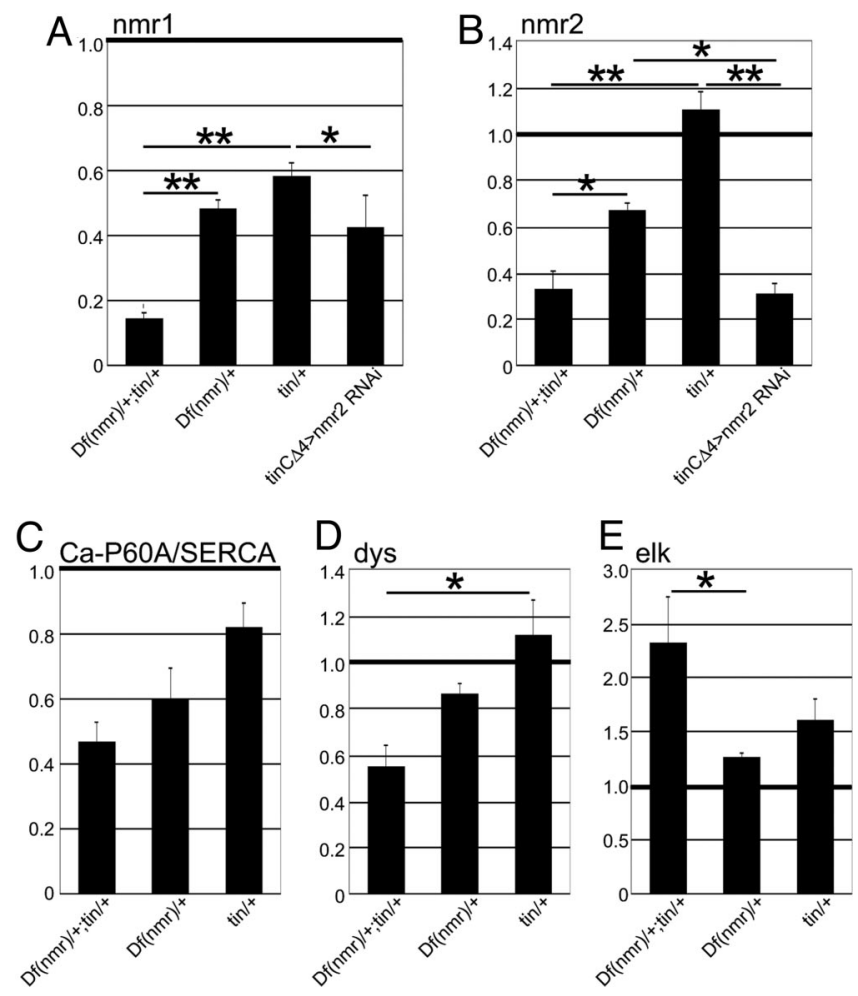

Fig. 4. Quantitative real-time $P C R$ on candidate genes in neuromancer, tinman mutants. Relative expression of $n m r 1, n m r 2$, Calcium ATPase at 60A (Ca-P60A; a SERCA homolog), dystrophin (dys), and eag-like $K^{+}$channel (elk) in 1-week-old adult hearts were standardized by $r p 49$ expression. Wild-type control $\left(w^{1118}\right)$ was set as one (indicated by thicker horizontal line). ( $A-D)$ The transheterozygotes of $n m r 1 \& 2$ deficiency [Df(2L)Exel6012] and tinman null mutants ( $\operatorname{tin}^{346} /+$ or tin ${ }^{E C 40}$ / + ), Df(nmr)/+;tin/+, showed lower expression than heterozygous Df(nmr)/+ or $\mathrm{tin} /+$ alone, indicating that $n m r 1$ itself, $n m r 2, C a-P 60 A$, and dys were positively regulated by $\mathrm{Nmr}$ and Tinman. The expression level of $n m r 2$ is also significantly reduced in the progeny of a cross between the heart-specific driver tinC $\Delta 4-\mathrm{Gal} 4$ and UAS-nmr2 RNAi line. $n m r 2$ expression in the $n m r 2$ knockdown flies is significantly reduced, compared to wild type $\left(w^{1118}\right)$ as well as to $\mathrm{Df}(n m r) /+$ or $\mathrm{tin} /+$. $(E)$ In contrast, the transheterozygotes showed higher level of elk expression indicating that elk may be negatively regulated by Nmr and Tinman. The normalized (to $w^{1118}$ ) average of independently prepared samples plus standard error is shown. We used 20 fly hearts for each sample (RNA extraction). Two samples were prepared for each genotype, thus tin/+ and Df(nmr)/+;tin/+ lanes are the normalized averages of four samples and $\mathrm{Df}(n m r) /+$ and tinc $\Delta 4>n m r 2$-RNAi the normalized averages of two samples each. For statistical analysis we used nonrepeated measures of ANOVA, then the differences were calculated between all combinations of each experimental group by Student-Newman-Keules test (parametric) shown in the following triangles; **,P<0.01; *, $P<0.05$.

Tbx20 has been widely studied in several model organisms, and the outcomes suggest a crucial developmental role of Tbx20 in cardiac specification and morphogenesis. Although a potential function of Tbx20 in the adult heart is likely (10), it has not been directly demonstrated. By using conditional and cardiac-specific manipulation, we found that $n m r / T b \times 20$ continues to have a regulatory role in the adult fly heart, and its depletion dramatically interferes with cardiac performance and disrupts contractile myofibrillar patterning. Since the cardiogenic gene $N k x 2-5$ is also required at later stages for proper cardiac function in vertebrates (29), as tinman and $n m r$ are in flies (this study; ref. 30 ), it is likely that Tbx20 also has an essential and conserved role in maintaining function and structural integrity in adult vertebrate hearts.

Interestingly, tinman and $n m r$ show a strong double heterozygous interaction in our Drosophila heart model that leads to severe arrhythmias, increased stress sensitivity, and muscle structure ab-

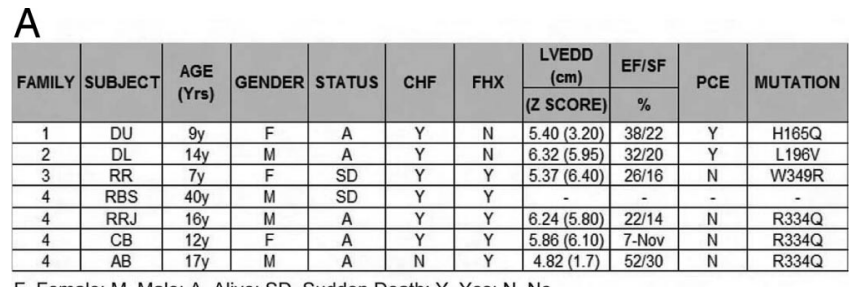

F, Female; M, Male; A, Alive; SD, Sudden Death; $Y$, Yes; N, No

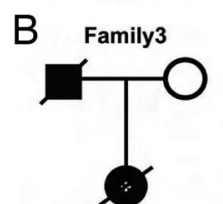

$\uparrow$ proband and carrier affected family members of the mutation W349R

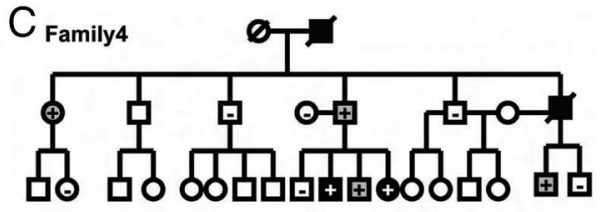

12 affected family members $\square \mathrm{O}$ unaffected family members

田( ) unaffected carrier of the mutation R334Q ('+' carrier of mutation and '-' negetive for the mutation).
Fig. 5. Tbx20 mutations in humans with cardiomyopathy. (A) Patient cohort details. Four types of Tbx20 mutations are identified from patients diagnosed with dilated cardiomyopathy (see SI Materials and Methods: Clinical data, for details). ( $B$ and $C$ ) Pedigrees of the families carrying the TBX20 mutation. ( $B$ ) In family 3, the 7-year old proband with the W349R mutation died of DCM. The father also died of acute idiopathic DCM (no sample available for genetic studies). (C) In family 4, the 16 year proband and his affected sister were detected with R334Q mutation. One of his unaffected brothers, his father, and some other extended family members are carriers of the mutation. In this family the grandfather and one of the uncles died suddenly of DCM.

normalities. In mice, Tbx20 and Nkx2-5 double heterozygotes also exhibit a synergistic interaction, which seem to be primarily confined to atrial defects (10). It is thus possible that the fly heart is sensitive to genetic interactions that are particularly relevant to the proper function of the mammalian atrium. These data imply that the fly heart can be used a discovery tool to identify synergistic genetic interaction that play a role in the mammalian heart in health and disease.

In an attempt to gage the predictive potential of the fly heart model in human heart function and dysfunction, we studied patients with DCM and performed genetic screening for TBX20 mutations in these patients. While mutations in NKX2-5 have been well-documented to be associated with congenital heart disease in humans $(16,42)$, heart defects potentially related to human TBX20 mutations have only recently been identified (this study; ref. 39). Here, we demonstrate three distinct TBX20 variants occurring in one sporadic and two familial cases of DCM. Clinically, the affected patients had classic features of DCM with associated rhythm disturbance, including, in some subjects, sudden death, heart failure, and arrhythmias. In the two families with TBX20 mutations, all subjects hosting the variants were afflicted with DCM. Additionally, multiple affected individuals had arrhythmias with rapid ventricular rhythm, while in others, atrioventricular (AV) block with bradycardia was notable. AV block was also noted in the sporadic case. The variants seen in these patients, which were not found in controls, disrupt conserved regions of the gene, including the T-box. These findings raise the possibility of an association of these variants with the disease. A demonstration, however, that the identified TBX20 mutations are causal in the development of the various observed heart disease phenotypes has to be achieved by functional studies. The identification of linkage to the TBX20 locus in large multigenerational families with heart disease would also strongly support a causal association. As predicted by the "final common pathway" hypothesis, the cytoskeleton and contractile apparatus interface, and its relationship to channel function, is likely to be affected by the TBX20 mutations (43). The finding of DCM, as well as the reduced penetrance that is seen in our 
study, is consistent with the findings reported by Kirk, et al. in human and murine studies of clinical phenotype in TBX20 mutants (39). Furthermore, studies of children with CHD identified two additional mutations in four individuals with various forms of ASD. These findings are also consistent with the report by Kirk, et al. (39). Our findings in the fly model, which led to our subsequent evaluation of humans with structural and myocardial disease, resulted in the suggestion of TBX20 as a promising candidate for a heart disease-causing gene that warrants further investigation and validation. These findings support the contention that the fly heart can serve as a promising and sensitive genetic predictor of gene candidates potentially involved in human heart disease.

\section{Materials and Methods}

Drosophila Stocks. $\operatorname{tin}^{346} / \mathrm{TM} 3-f t z l a c Z(2), \operatorname{tin}^{E C 40} / \mathrm{TM} 3, \mathrm{Df}(3 \mathrm{R}) \operatorname{tin}^{G C 14}$ (1), Df(3L)DocA (37), nmr1614, nmr1210 (13), Df(2L)Exel6012 (38), also indicated as $\mathrm{Df}(n m r)$. UAS-Gal4 system was used as in (45). Gal4 and UAS lines used: twi-Gal4 (twi >; ref. 44), 24B-Gal4 (24B >; ref. 45), twi-Gal4;24B-Gal4 (twi24B>; ref. 46), tinCs4-Gal4 (47), Dmef2-Gal4 (48), UAS-tin (49), UAS-nmr2RNAi, UAS-nmr1, and UAS-nmr2 (13).

1. Bodmer $R$ (1993) The gene tinman is required for specification of the heart and visceral muscles in Drosophila. Development 118:719-729.

2. Azpiazu N, Frasch M (1993) Tinman and bagpipe: Two homeo box genes that determine cell fates in the dorsal mesoderm of Drosophila. Genes Dev. 7:1325-1340.

3. Cripps RM, Olson EN (2002) Control of cardiac development by an evolutionarily conserved transcriptional network. Dev Biol 246:14-28.

4. Qian L, Liu J, Bodmer R (2008) Heart development in Drosophila. Advances in Developmental Biology: Cardiac Development, ed R Bodmer (Elsevier Publishing, New York, NY), pp. 1-29.

5. Zaffran S, Frasch M (2002) Early signals in cardiac development. Circ Res 91:457-469.

6. Bier E, Bodmer R (2004) Drosophila, an emerging model for cardiac disease. Gene 342:1-11.

7. Plageman TF, Jr., Yutzey KE (2004) Differential expression and function of Tbx5 and Tbx20 in cardiac development. J Biol Chem 279:19026-19034.

8. Cai CL, et al. (2005) T-box genes coordinate regional rates of proliferation and regional specification during cardiogenesis. Development 132:2475-2487.

9. Singh MK, et al. (2005) Tbx20 is essential for cardiac chamber differentiation and repression of Tbx2. Development 132:2697-2707.

10. Stennard FA, et al. (2005) Murine T-box transcription factor Tbx20 acts as a repressor during heart development, and is essential for adult heart integrity, function and adaptation. Development 132:2451-2462

11. Takeuchi JK, et al. (2005) Tbx20 dose-dependently regulates transcription factor networks required for mouse heart and motoneuron development. Development 132:2463-2474.

12. Miskolczi-McCallum CM, et al. (2005) The Drosophila melanogaster T-box genes midline and $\mathrm{H} 15$ are conserved regulators of heart development. Dev Biol 278:459-472.

13. Qian L, Liu J, Bodmer R (2005) Neuromancer Tbx20-related genes (H15/midline) promote cell fate specification and morphogenesis of the Drosophila heart. Dev Bio/ 279:509-524.

14. Reim I, Mohler JP, Frasch $\mathrm{M}$ (2005) Tbx20-related genes, mid and H15, are required for tinman expression, proper patterning, and normal differentiation of cardioblasts in Drosophila. Mech Dev 122:1056-1069.

15. Garg V, et al. (2003) GATA4 mutations cause human congenital heart defects and reveal an interaction with TBX5. Nature 424:443-447.

16. Schott JJ, et al. (1998) Congenital heart disease caused by mutations in the transcription factor NKX2-5. Science 281:108-111.

17. Basson CT, et al (1997) Mutations in human TBX5 [corrected] cause limb and cardiac malformation in Holt-Oram syndrome. Nat Genet 15:30-35.

18. Li QY, et al., (1997) Holt-Oram syndrome is caused by mutations in TBX5, a member of the Brachyury (T) gene family. Nat Genet 15:21-29.

19. Prall OW, Elliott DA, Harvey RP (2002) Developmental paradigms in heart disease: Insights from tinman. Ann Med 34:148-156.

20. Brown DD, et al (2005) Tbx5 and Tbx20 act synergistically to control vertebrate heart morphogenesis. Development 132:553-563.

21. Stennard FA, etal (2003) Cardiac T-box factor Tbx20 directly interacts with Nkx2-5, GATA4, and GATA5 in regulation of gene expression in the developing heart. Dev Biol 262:206224.

22. Feany MB, Bender WW (2000) A Drosophila model of Parkinson's disease. Nature 404:394398.

23. Muqit MM, Feany MB (2002) Modelling neurodegenerative diseases in Drosophila: A fruitful approach? Nat Rev Neurosci 3:237-243.

24. Wessells RJ, et al (2004) Insulin regulation of heart function in aging fruit flies. Nat Genet 36:1275-1281.

25. Wolf MJ, et al (2006) Drosophila as a model for the identification of genes causing adult human heart disease. Proc Natl Acad Sci USA 103:1394-1399.
Electrical Pacing. The electrical pacing was conducted as previously described ( 24 31). Briefly, 50-100 flies were paced with a square wave stimulator at $40 \mathrm{~V}$ and 6 $\mathrm{Hz}$ for $30 \mathrm{~s}$ and scored for heart arrest/fibrillation rate.

Image Analysis and M-mode Traces on Semiintact Preparations. Semiintact heart preparations for image analysis were as in Ocorr, et al. (26). M-modes from movies were generated using Simple $\mathrm{PCl}$ software and MatLab-based image analysis (26).

Quantitative Real-Time PCR. RNA was extracted from 20 1-week old hearts for each genotype and subjected to qRT-PCR as described in SI Text.

Patient Evaluation. Patients were evaluated by chest radiography, electrocardiography, echocardiography, and MRI. Left ventricular size and function were evaluated by M-mode and two-dimensional Doppler and color Doppler echocardiographic images. Further details are described in SI Text.

ACKNOWLEDGMENTS. We thank the Bloomington stock center and Devel opmental Studies Hybridoma Bank for sending fly-stocks and antibodies. L.Q. and J. L. were supported by a predoctoral fellowship from the American Heart Association. This work was funded by grants from National Heart, Lung, and Blood Institute of the National Institutes of Health to J.A.T and R.B.

26. Ocorr K, et al (2007) KCNQ potassium channel mutations cause cardiac arrhythmias in Drosophila that mimic the effects of aging. Proc Natl Acad Sci USA 104:3943-3948.

27. Lyons I, et al. (1995) Myogenic and morphogenetic defects in the heart tubes of murine embryos lacking the homeo box gene Nkx2-5. Genes Dev 9:1654-1666.

28. Tanaka M., et al. (2001) Nkx2.5 and Nkx2.6, homologs of Drosophila tinman, are required for development of the pharynx. Mol Cell Biol 21:4391-4398.

29. Pashmforoush M., et al. (2004) Nkx2-5 pathways and congenital heart disease; loss of ventricular myocyte lineage specification leads to progressive cardiomyopathy and complete heart block. Cell 117:373-386.

30. Zaffran S, et al. (2006) Cardioblast-intrinsic Tinman activity controls proper diversification and differentiation of myocardial cells in Drosophila. Development 133:4073-4083.

31. Wessells RJ, Bodmer R (2004) Screening assays for heart function mutants in Drosophila. Biotechniques 37:58-60, 62, 64 passim.

32. McGuire SE, Mao Z, Davis RL (2004) Spatiotemporal gene expression targeting with the TARGET and gene-switch systems in Drosophila. SCi STKE 2004:I6.

33. Saide JD, et al. (1989) Characterization of components of Z-bands in the fibrillar flight muscle of Drosophila melanogaster. J Cell Biol 109:2157-2167.

34. Molina MR, Cripps RM (2001) Ostia, the inflow tracts of the Drosophila heart, develop from a genetically distinct subset of cardial cells. Mech Dev 109:51-59.

35. Monier B, et al. (2005) Steroid-dependent modification of Hox function drives myocyte reprogramming in the Drosophila heart. Development 132:5283-5293.

36. Bruneau BG, et al. (2001) A murine model of Holt-Oram syndrome defines roles of the T-box transcription factor Tbx5 in cardiogenesis and disease. Cell 106:709-721.

37. Reim I, Lee HH, Frasch M (2003) The T-box-encoding Dorsocross genes function in amnioserosa development and the patterning of the dorsolateral germ band downstream of Dpp. Development 130:3187-3204.

38. Taghli-Lamallem O, et al. (2008) Dystrophin-deficiency in Drosophila reduces lifespan and causes a dilated cardiomyopathy phenotype. Aging Cell 7: 237-249.

39. Kirk EP, et al. (2007) Mutations in cardiac T-box factor gene TBX20 are associated with diverse cardiac pathologies, including defects of septation and valvulogenesis and cardiomyopathy. Am J Hum Genet 81:280-291.

40. Priori SG, etal. (1999) Low penetrance in the long QT syndrome: Clinical impact. Circulation 99:529-533.

41. Dalal $D$, et al. (2006) Penetrance of mutations in plakophilin-2 among families with arrhythmogenic right ventricular dysplasia/cardiomyopathy. J Am Coll Cardiol 48: 1416-1424.

42. Benson DW, et al. (1999) Mutations in the cardiac transcription factor NKX2.5 affect diverse cardiac developmental pathways. J Clin Invest 104:1567-1573.

43. Bowles NE, Bowles KR, Towbin JA (2000) The "final common pathway" hypothesis and inherited cardiovascular disease. The role of cytoskeletal proteins in dilated cardiomyopathy. Herz 25:168-175.

44. Greig S, Akam M (1993) Homeotic genes autonomously specify one aspect of pattern in the Drosophila mesoderm. Nature 362:630-632.

45. Brand AH, Perrimon N (1993) Targeted gene expression as a means of altering cell fates and generating dominant phenotypes. Development 118:401-415.

46. Lockwood WK, Bodmer R (2002) The patterns of wingless, decapentaplegic, and tinman position the Drosophila heart. Mech Dev 114:13-26.

47. Lo PC, Frasch M (2001) A role for the COUP-TF-related gene seven-up in the diversification of cardioblast identities in the dorsal vessel of Drosophila. Mech Dev 104:49-60.

48. Bour BA, et al. (1995) Drosophila MEF2, a transcription factor that is essential for myogenesis. Genes Dev 9:730-741.

49. Ranganayakulu G, et al. (1998) Divergent roles for NK-2 class homeobox genes in cardiogenesis in flies and mice. Development 125:3037-3048. 